This is an accepted manuscript to the 2019 IEEE International Conference on Prognostics and Health Management

The final manuscript can be found under the following link:

https://ieeexplore.ieee.org/abstract/document/8819397

(C) 2019 IEEE. Personal use of this material is permitted. Permission from IEEE must be obtained for all other uses, in any current or future media, including reprinting/republishing this material for advertising or promotional purposes, creating new collective works, for resale or redistribution to servers or lists, or reuse of any copyrighted component of this work in other works 


\section{Formulation and Solution for the Predictive Maintenance Integrated Job Shop Scheduling Problem}

\author{
Simon Zhai \\ Institute for Machine Tools and Industrial \\ Management \\ Department of Mechanical Engineering \\ Technical University of Munich \\ Garching, Germany \\ simon.zhai@iwb.mw.tum.de
}

\author{
Alexander Riess \\ Institute for Machine Tools and Industrial \\ Management \\ Department of Mechanical Engineering \\ Technical University of Munich \\ Garching, Germany \\ alexander.riess@tum.de
}

\author{
Gunther Reinhart \\ Institute for Machine Tools and Industrial \\ Management \\ Department of Mechanical Engineering \\ Technical University of Munich \\ Garching, Germany \\ gunther.reinhart@iwb.mw.tum.de
}

\begin{abstract}
Predictive Maintenance has gained a lot of attention in recent years due to the development of improved sensors and intelligent algorithms. These allow for monitoring the health condition of production machinery and predict its future deterioration. In order to generate added value for industrial use cases, two more steps are required: considering the machine's time-varying operational conditions and integrating its dependent deterioration prediction in a holistic scheduling approach. This publication identifies a shortage of deterioration estimation frameworks under time-varying operational conditions as well as a lack of Predictive Maintenance integrated scheduling problems in the literature. Subsequently, a new conceptual framework to model future machine deterioration under time-varying operational conditions and its application in production scheduling is introduced. The Operation Specific Stress Equivalent (OSSE) represents the load of a future production job on the machine and supports a general formulation of the maintenance integrated job shop scheduling problem (MIJSSP). This formulation is presented together with benchmark instances and corresponding sample data. Finally, the formulation is tested with the help of a genetic algorithm that illustrates the potential of using new objective functions for decision support, such as the Reliability Weighted Makespan $C_{\max }{ }^{R}$.
\end{abstract}

Keywords- Predictive Maintenance, Integrated Scheduling, Optimization, Decision Support

\section{INTRODUCTION}

Within Industrie 4.0, Predictive Maintenance (PdM) is considered to be one of the most promising innovations. By embedding smart, connected technology within machines or production sites, it is possible to monitor the health of a system through ongoing measurements, apply analytics, predict its future deterioration and remaining useful life (RUL) [1]. Over $80 \%$ of surveyed manufacturing companies are aware of the enormous upside potential of PdM and are currently elaborating their opportunities intensively [2]. The digitalization and its increasing flood of data confronts manufacturing companies - especially Small and Mediumsized Enterprises (SMEs) - with strategic challenges. Despite of being assessed as highly relevant in the next two to five years, the capability and applicability of PdM for industrial machinery today is still considered low [3].

While condition monitoring as well as the associated pattern detection and failure prediction are widely discussed in the literature and new technologies have led to significant improvements in research over the past years, the essential step is the application of the obtained knowledge in everyday manufacturing and maintenance operation. The job shop is a widely applied manufacturing process model in a multitude of industries and thus, a lot of scheduling approaches for job shop production exist. However, few approaches have been proposed for combining the so-called Job Shop Scheduling Problem (JSSP) and maintenance planning activities while taking current and future machine conditions into account $[4,5]$. For the success of PdM in the industry, it is thus important to extend the JSSP research by integrating machine deterioration and developing a combined production and maintenance scheduling model. With integrated scheduling, the deterioration of the machines - and thus, the maintenance point in time - can be controlled and machine failures can be avoided. This publication thus proposes a novel approach to integrate production and maintenance scheduling. This publication aims at providing a new conceptual framework for maintenance integrated production scheduling.

First, a literature review locates the research gap. Second, the model of Operation Specific Stress Equivalents (OSSEs) is introduced. Afterwards, the Maintenance Integrated Job Shop Scheduling Problem (MIJSSP) is formulated and a solution and simulation approach are presented. Finally, the approach is validated, and the conclusion shows paths for future research.

Funded by the European Union's Horizon 2020 program under the Grant Agreement No. 768575

978-1-5386-8357-6/19/\$31.00 (C)2019 IEEE 


\section{LITERATURE REVIEW}

The literature review examines the deterioration estimation and RUL prediction for industrial machinery under constant and time-varying operational conditions, with the focus on latter. Furthermore, it highlights the state-of-the-art research of holistic scheduling approaches integrating production and maintenance jobs as well as machine deterioration.

\section{A. Condition Monitoring and RUL prediction}

Through artificial intelligence and big data analytics it is possible to assess current and predict future machine conditions. In this context, $\mathrm{PdM}$ and prognostics and health management (PHM) research aim at estimating a machine's RUL and its existent or subsequently emerging risk of failure [6]. This section shortly introduces main concepts of RULmodelling, with the focus on work that permit maintenanceintegrated production scheduling. According to [7] the current techniques for RUL estimation can be roughly classified into physical-based approaches, data-driven approaches and their combinations, i.e. hybrid approaches. As [7] state that "complex systems [...] [are too] complicated to map their precise physics to their exact failure mechanisms, thus datadriven approaches are good alternatives to accomplish the prognostic tasks", this section focuses data driven approaches. The reader shall be referred to [8] and [9] for a review of physical-based models for machine components.

For complex systems as machine tools used in job shop production, many data-driven approaches for RUL estimation employ data fitting methods including machine learning and statistics-based methods to model the system deterioration process. According to [7], "existing methods have broadly presented two ways to describe the uncertainty in a degradation model. One is using probabilistic methods, in which the deterioration phenomenon is considered to have certain random behavior which is often characterized by probability laws, e.g., normal distribution, or by stochastic models, e.g. Wiener, Gamma and inverse Gaussian processes. The other can be referred to as parameter updating strategies, which updates the model's parameter by newly available data acquired from currently functioning individuals". A concise overview of different data-driven approaches to RUL modelling of the popular C-MAPSS data set is given by [10]. Three general categories are found: functional mapping between inputs and RUL, mappings between a health index and RUL and matching. In those categories, the authors of [10] identify, among others, artificial neural networks, fuzzy rules and regression techniques capable of modelling RUL. For an overview of different invariant modelling techniques, the reader shall be referred to the review of [1].

Variant approaches: As noted by [11], "to date, the vast majority of failure models have assumed that the prevailing environmental conditions are temporally invariant or have no effect on degradation [...] and assume that the operational conditions are invariant, or not affecting the deterioration process". This is not always true in engineering applications, especially in the context of job shop production. The operational conditions usually vary between different operations, and in most cases, and have different effects on the process of deterioration. Therefore, it is necessary to develop operation specific and schedule dependent inputs for the scheduling process. A framework for predicting the RUL of a machine under time-varying operating conditions is provided by [12]. First, changing, but piecewise constant operating conditions are assumed, then a more complex case with stochastic future operational conditions is examined. The underlying deterioration process is modeled as a Brownian motion, which evolves responding to the operating conditions and the unit's RUL is predicted through a Bayesian technique. In the end, simulation experiments and a case study for ball bearings are conducted. A method based on probabilistic and stochastic approaches to estimate system RUL for a periodically monitored degradation process with dynamic timevarying operational conditions is presented by [7]. It is assumed that the transition between different operational conditions plays the most important role in affecting the degradation process. These operational conditions are modelled with the help of a discrete-time Markov chain.

The same requirement applies to most engineering applications, as the deterioration is affected by the environmental and operational conditions in which the components are used [11]. For example, when a machine tool rotates at higher operational speeds, it generates vibration amplitudes that are higher than those generated at lower speeds. The time-varying nature of the environment or operating conditions effect the degradation. Thus, it is necessary to convey the operation specific and schedule dependent deterioration estimation to the job shop production as inputs for the scheduling process.

\section{B. Integrated Production and Maintenance Scheduling}

The integration of maintenance and production scheduling starts to draw researcher's interest, since machine breakdowns and subsequent production delays lead to high cost in the industry [13]. Having been treated independently in the past, a lot of separate models for both tasks have been presented. According to [4], it is widely believed that independent treatment of scheduling of production and maintenance will provide suboptimal solutions, due to the fact that they are interrelated. Proper understanding of this dependency will open a new avenue for more integrated models, and result in significant savings in operational cost and improved efficiency for any production system. [4] and [5] both review the literature on models addressing different aspects of integration in the areas of maintenance production scheduling.

Integrated preventive maintenance (PM) scheduling: The JSSP with sequence dependent setup times and different PM policies, i.e. scheduled maintenance to minimize the makespan, were considered by [14]. The makespan describes the timespan between the first job entering and last job exiting the production system [15]. Two maintenance policies and four metaheuristics based on simulated annealing and genetic algorithms (GA) were employed to solve the problem. An integrated preventive maintenance planning and production scheduling problem for a single machine with the objective to minimize the expected total weighted tardiness were first formulated by [16]. Another model for integrating production scheduling and maintenance 
planning in the JSSP was developed by [17]. It is assumed that PM is imperfect and cannot restore the machine to an as good as new condition, while replacement is considered as perfect maintenance. The objective was to optimize the total profit, which was modelled as production values minus production costs, maintenance costs including PM and replacement costs, as well as tardiness costs. A new heuristic is proposed by [18] to solve the joint JSSP of production and maintenance actions by extending the standard GA with the idea of enhancing its random character by guiding the steps in a logical procedure. In [19], the integrated planning of production, maintenance and quality in deteriorating systems is investigated.

Scheduling with job specific deterioration: The authors of [20] and [21] were the first to present the integrated JSSP in an job specific manner. While [20] introduced the JSSP with job specific deterioration and $\mathrm{PdM}$ and presented a heuristic to solve it, [21] divided the scheduling problem in three parts, namely the production scheduling problem, the PHM problem, and the PdM scheduling problem. While the production scheduling problem made the common assumptions for a single machine, the PHM problem formulation assumed that the machine's condition is continuously monitored. As on a single multifunctional machine different jobs with different machine parameters are processed, a job-depending deterioration process was proposed. Resembling the notation of [21], the RUL model is formulated in a job-specific manner. It is assumed that the $R U L_{i k}$ of a machine $M_{k}$ processing job $J_{i}$ with processing time $p_{i k}$ represents the time during which the machine is able to produce the job without breakdown. The $R U L_{i k}$ value is considered to be known, certain and of linear characteristic. Based on the RUL information one can determine a deterioration ratio. For an operation $O_{i k}$ on machine $M_{k}$, the deterioration progress $\delta_{i k}=p_{i k} / R U L_{i k}$ is assumed.

\section{Research Gap}

In conclusion, the concept of condition monitoring and predicting the remaining operation life of a machine has been successfully established in the literature and has started to be applied in the industry [22]. However, the found work emphasizes the further need for additional research regarding the interpretation and application on machine condition information in the actual production scheduling processes. For this purpose, existing literature is content with exploring the JSSP and PdM according to machine deterioration or failure independently. This publication aims at developing a holistic framework that allows for an integration of production scheduling, machine deterioration estimation and machine breakdown prediction. Furthermore, the need of modelling operation specific deterioration or stresses was identified in order to enable such integrated scheduling, which in turn enables the implementation of more profound PdM strategies (see Chapter III). This publication thus explores a possible extension to the existing scheduling problem formulations and proposes the new Maintenance Integrated Job Shop Scheduling Problem (MIJSSP), which considers machine deterioration, machine breakdowns, as well as operation specific stress equivalents which model invariant machine conditions. A suitable solution method for the new MIJSSP is presented to explore the potential of the new problem formulation and is tested in a developed simulation environment which allows for a quantitative comparison.

\section{The Model of Operation SPECIFIC StREsS EquivalentS}

As deduced from the literature review, an integrated scheduling model requires information about the probability of an unplanned machine breakdown. Therefore, the machine's historic deterioration as well as its future stress load have to be taken into account when modeling the machine's health $[20$, 21]. For this purpose, the proposed framework distinguishes an operation-focused deterioration estimation and a machinefocused failure probability prediction.

\section{A. Deterioration Estimation}

As different operations impose different levels of stress on a machine, the estimated time the machine is able to produce within a given deterioration limit is operation specific. The supplied information of a PHM module, namely $R U L_{i k}$ of a machine $M_{k}$ processing operation $O_{i}$ is assumed to be known and certain for modelling the machine condition. $R U L_{i k}$ defines the point in time the machine $M_{k}$ processing only operation $O_{i}$ is fully deteriorated and will most likely fail. Given a certain processing time $p_{i}$ the level of deterioration imposed on a machine by an operation $O_{i}$ can be determined as its share in the machine's $R U L_{i k}[20,21]$. Consequently, the Operation Specific Stress Equivalent (OSSE) is defined as

$$
\delta_{i k}=\frac{p_{i}}{R U L_{i k}}
$$

Table 1 illustrates this assumption. For intuitiveness and comparability, it is suggested that the PHM module provides the $R U L_{i k}$ of an operation $O_{i}$ based on the machine's as good as new condition. This assumption allows for direct comparability of the different RULs and OSSEs and simplifies the deterioration estimation of the machine to

$$
\Delta_{k}\left(t_{1}\right)=\Delta_{k}\left(t_{0}\right)+\Delta_{k}\left(t_{1}-t_{0}\right)=\Delta_{k}\left(t_{0}\right)+\delta_{i k}
$$

with $\Delta_{k}\left(t_{1}\right)$ being the cumulative deterioration of machine $\mathrm{M}_{k}$ at time $t_{1}$, i.e. after running the operation. Fig. 1 displays an example. This allows the OSSE $\delta_{i k} \in[0 ; 1]$ to be interpreted as the share of deterioration potential of an operation $O_{i}$ running on a machine $M_{k}$. Put more simply, operations imposing higher stress are represented by a higher $\delta_{i k}$, which originates either from a longer processing time $p_{i}$ or a shorter $R U L_{i k}$. Consequently, the deterioration state of a machine $M_{k}$ at a point in time $t$ is given by $\Delta k(t)$, where 0 denotes an as good as new condition and 1 denotes the depletion of the characteristic life, up to which the machine is expected to have failed $63.2 \%$ of the time [23].

\begin{tabular}{lccc}
\hline Operation & $p_{i}$ & $R U L_{i k}$ & OSSE $\delta_{i k}$ \\
\hline $\mathrm{O}_{1}$ & 25 & 500 & 0.05 \\
$\mathrm{O}_{2}$ & 40 & 160 & 0.25 \\
$\mathrm{O}_{3}$ & 10 & 100 & 0.10 \\
\hline
\end{tabular}



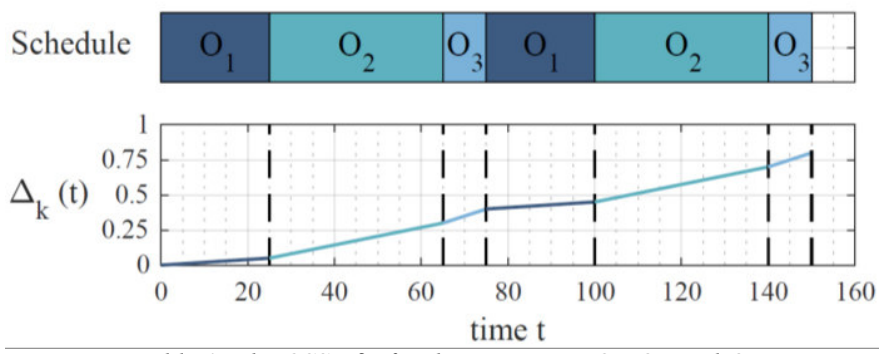

Table 1: The OSSE $\delta_{i k}$ for the operations $O_{1}, O_{2}$, and $O_{3}$

Fig. 1 The deterioration estimation for O1-O2-O3-O1-O2-O3.

Fig. 1 illustrates $\Delta_{k}(t)$ for an exemplary operations schedule O1-O2-O3-O1-O2-O3, where $\Delta_{k}(t=150)=\delta_{1 k}+\delta_{2 k}+\delta_{3 k}+\delta_{1 k}+\delta_{2 k}+\delta_{3 k}=0.80$.

\section{B. Reliability Prediction}

The previous chapter introduced that a machine follows an operation sequence dependent, yet predictable deterioration process. However, it is not the machine's estimated deterioration but its actual unavailability that is of primary interest for the scheduling process. A lot of research in the field of unplanned machine failures has been conducted and a machine's reliability can be measured by a variety of parameters, one of the most used being the failure rate $\lambda$ [23]. The failure rate is the frequency in which a machine fails, expressed in number of failures per unit of time.

For modelling the probability and distribution of those failures, the Weibull distribution is particularly useful and commonly applied in engineering reliability research [24]. The so-called two-parameter Weibull is a general mathematical distribution which, by adjustment of the shape parameter $\beta$ and the scale parameter $\eta$, allows for modelling a wide range of failure distribution characteristics of different machines and systems. Its probability density function $f(t)$ is denoted as follows:

$$
f(t)=\frac{\beta}{\eta} \cdot\left(\frac{t}{\eta}\right)^{\beta-1} \cdot e^{-\left(\frac{t}{\eta}\right)^{\beta}}
$$

The cumulative distribution function sums up the failure probabilities before a certain point in time $t$ and can thus be interpreted as the failure probability function $F(t)$ which describes the likelihood that a machine has failed before this point in time. In reverse, $R(t)$ can be interpreted as the reliability function which returns the probability of a machine operating without failure until a point in time $t$. It is defined as

$$
R(t)=1-F(t)=e^{-\left(\frac{t}{\eta}\right)^{\beta}}
$$

The failure probability function and the reliability function further allow for the calculation of the hazard function $h(t)$ of the machine. [24] points out that the hazard function in engineering applications is often described as the failure rate $\lambda(t)$ at a given point in time $t$ and can be expressed as

$$
\lambda(t)=\frac{f(t)}{R(t)}=\frac{\beta}{\eta} \cdot\left(\frac{t}{\eta}\right)^{\beta-1}
$$

For further reliability predictions of a production machine in a running operation, especially constant/random failure rates $(\beta=1)$ as well as the wear out failure rates $(\beta>1)$ at the end of a machine' life are of interest.

Mathematically, [24] denotes the deterioration progress as ratio of the operational time $t$ passed and the remaining operational life time $\eta$. Following the deterioration estimation (cf. III.A), for a specific operation $O_{i}$, the operational time passed $t$ equals the operation processing time $p_{i}$ and the remaining operational life time $\eta$ is identical with the $R U L_{i k}$ provided by the PHM module. According to the introduced OSSE $\delta_{i k}$, this yields:

$$
\frac{t}{\eta}=\frac{p_{i}}{R U L_{i k}}=\delta_{i k}
$$

The failure rate $\lambda(t)$ has to follow a discontinuous shape as it is dependent of the specific stress currently imposed by an operation. Subsequently, the total reliability $R(t)$ of a production machine is a composed, but continuous and monotonically decreasing function as shown in Fig. 2 and Fig. 3 for different values of the shape parameter $\beta$.

As each operation imposes a specific level of deterioration $\delta_{\mathrm{ik}}$ to a machine $M_{k}$, it is suggested that the deterioration progress is a composed function of the imposed OSSEs on a machine, which can be denoted as the $\Sigma \delta_{i k}$ or the machine deterioration $\Delta \Delta_{k}$. In conclusion:

$$
\frac{t}{\eta}=\sum_{i} \frac{p_{i}}{R U L_{i k}}=\sum_{i} \delta_{i k}=\Delta_{k}(t)
$$

This then yields the $\beta$-parametric reliability and failure rate functions, which allow for operation specific failure probability prediction:

$$
\begin{gathered}
f(t)=\frac{\beta}{R U L_{i k}} \cdot \Delta_{k}(t)^{\beta-1} \cdot e^{-\Delta_{k}(t)^{\beta}} \\
R(t)=1-F(t)=e^{-\Delta_{k}(t)^{\beta}} \\
\lambda_{i}(t)=\frac{f(t)}{R(t)}=\frac{\beta}{R U L_{i k}} \cdot \Delta_{k}(t)^{\beta-1}
\end{gathered}
$$

For the exemplary operations schedule $\mathrm{O}_{1}-\mathrm{O}_{2}-\mathrm{O}_{3} \mathrm{O}_{1^{-}} \mathrm{O}_{2}-\mathrm{O}_{3}$ (cf. Table 3 for parameter values), Fig. 2 depicts the resulting $\lambda_{i}(t)$ and $R(t)$ for a shape parameter of $\beta=1$, where $R(t=150) \approx 0.40$. While a shape parameter of $\beta=1$ leads to $\lambda(t)=1 / R U L_{i k}$ and thus a constant failure rate; $\beta>1$ yields increasing failures rates over time. This is illustrated in Fig. 3, where $R(t=150) \approx 0.45$.

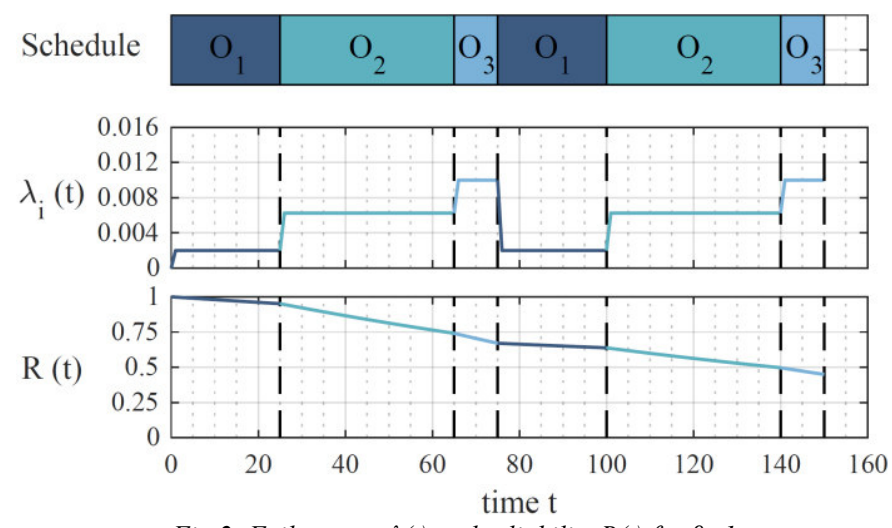

Fig 2: Failure rate $\lambda(t)$ and reliability $R(t)$ for $\beta=1$ 
Schedule
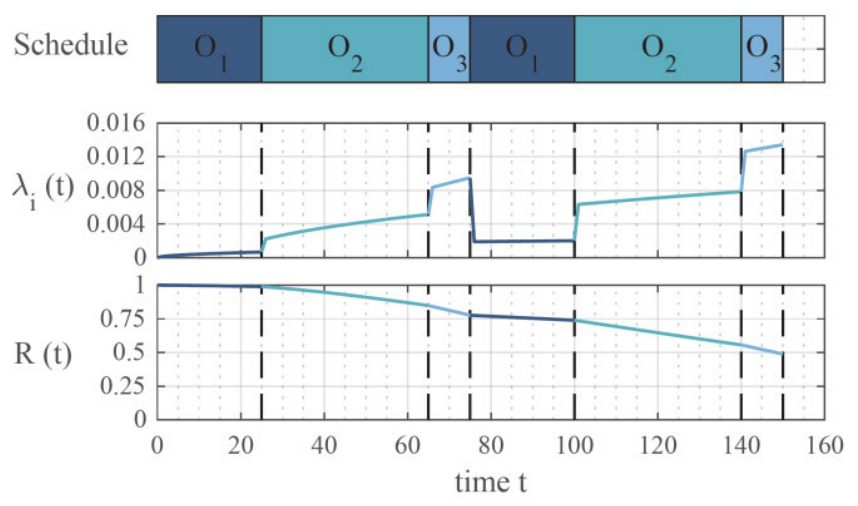

Fig 3: Failure rate $\lambda(t)$ and reliability $R(t)$ for $\beta=1.5$

The figures clearly show how different values for $\beta$ affect the reliability prediction. E.g. note that in Fig. 3, the second instances of the operations lead to higher values of $\lambda$, and thus realistically models the wear-out behavior of a machine.

\section{The MAINTENANCE INTEGRATED JoB SHOP SCHEDULING PROBLEM}

The introduction of the OSSE as well as the reliability and hazard function allow for the formulation of a new holistic maintenance integrated job shop scheduling problem (MIJSSP). While several sets of benchmark instances for the JSSP can be found in the literature, it is required to complement them with maintenance and failure information for future research in the field of Predictive Maintenance scheduling.

\section{A. Adoption of Literature Benchmark Instances}

A variety of JSSP benchmark instances are available in the literature for comparing the performance of different production scheduling models [25-27] as summarized by [28]. It is suggested above that an OSSE is needed to predict a machine's deterioration state and thus estimate its failure probability. Although the data for famous JSSP benchmark instances in the literature were partially generated randomly [29], this approach doesn't apply fully to the generation of the OSSEs for a benchmark instance. They have a direct impact on the machine's deterioration state, which again strongly influences the integrated production and maintenance scheduling. Therefore, a realistic ratio of a machine's deterioration to its productivity is desired. To estimate a reasonable range for the OSSEs given a specific problem instance, four additional parameters are required for extending any traditional JSSP: 1) average number of failures per machine during the makespan, 2) duration of a failure, 3) number of possible maintenance actions per machine during the makespan, 4) duration of a maintenance action.

The parameter ranges can either be drawn from literature findings, expert knowledge or derived from real life industry scenarios. Together with the stated number of operations and the respective operation times, these figures allow the calculation of the required average OSSE for the given JSSP. The particular OSSE values can then be drawn randomly for each operation within defined limits around the calculated average, e.g. with a normal or uniform distribution depending on the desired problem characteristics.

\section{B. Exemplary Adoption of the ft10-JSSP to a MIJSSP}

Hereafter the process of refining a given JSSP from the literature with maintenance and failure information is shown using the example of the $f t 10$ instance [25], one of the bestknown JSSP benchmarks. The 10x10 problem instance is given in the Taillard-Representation [29], consisting of two matrices: Table 2 shows the operation sequence - the order a job is visiting the different machines - and Table 3 depicts the respective operation times. With the assumptions shown in Table 4, that according to expert knowledge resemble a real industrial use case, the MIJSSP derived from the $f t 10$ instance is estimated to have up to five machine failures in total (on average 0.5 failures per machine), each lasting 100 time units. In addition, up to one maintenance interval of 25 time units per machine is available, resetting its deterioration to an as good as new condition. With the help of the reliability prediction (cf. section III.B) and multiple simulation runs, these assumptions yield the opportunity to define the OSSEs in a way that resembles the desired deterioration and thus failure probabilities of the machines. By extending the $f t 10$ as shown in Table 5, the JSSP has now become a MIJSSP and can be scheduled and benchmarked with different new and holistic scheduling models. The same process can be applied to extend industry JSSPs for the consideration of deterioration estimation and failure prediction. This allows for holistic PdM scheduling and the estimation of its potential for a company or industry.

Table 2: Operation sequence of the ft10 instance [25]

\begin{tabular}{lcccccccccc}
\hline Job & $O_{i 1}$ & $O_{i 2}$ & $O_{i 3}$ & $O_{i 4}$ & $O_{i 5}$ & $O_{i 6}$ & $O_{i 7}$ & $O_{i 8}$ & $O_{i 9}$ & $O_{i 10}$ \\
\hline Job 1 & 1 & 2 & 3 & 4 & 5 & 6 & 7 & 8 & 9 & 10 \\
Job 2 & 1 & 3 & 5 & 10 & 4 & 2 & 7 & 6 & 8 & 9 \\
Job 3 & 2 & 1 & 4 & 3 & 9 & 6 & 8 & 7 & 10 & 5 \\
Job 4 & 2 & 3 & 1 & 5 & 7 & 9 & 8 & 4 & 10 & 6 \\
Job 5 & 3 & 1 & 2 & 6 & 4 & 5 & 9 & 8 & 10 & 7 \\
Job 6 & 3 & 2 & 6 & 4 & 9 & 10 & 1 & 7 & 5 & 8 \\
Job 7 & 2 & 1 & 4 & 3 & 7 & 6 & 10 & 9 & 8 & 5 \\
Job 8 & 3 & 1 & 2 & 6 & 5 & 7 & 9 & 10 & 8 & 4 \\
Job 9 & 1 & 2 & 4 & 6 & 3 & 10 & 7 & 8 & 5 & 9 \\
Job 10 & 2 & 1 & 3 & 7 & 9 & 10 & 6 & 4 & 5 & 8 \\
\hline
\end{tabular}

Table 3: Operation times of the ft10 instance [25]

\begin{tabular}{lcccccccccc}
\hline Job & $p_{i 1}$ & $p_{i 2}$ & $p_{i 3}$ & $p_{i 4}$ & $p_{i 5}$ & $p_{i 6}$ & $p_{i 7}$ & $p_{i 8}$ & $p_{i 9}$ & $p_{i l 0}$ \\
\hline Job 1 & 29 & 78 & 9 & 36 & 49 & 11 & 62 & 56 & 44 & 21 \\
Job 2 & 43 & 90 & 75 & 11 & 69 & 28 & 46 & 46 & 72 & 30 \\
Job 3 & 91 & 85 & 39 & 74 & 90 & 10 & 12 & 89 & 45 & 33 \\
Job 4 & 81 & 95 & 71 & 99 & 9 & 52 & 85 & 98 & 22 & 43 \\
Job 5 & 14 & 6 & 22 & 61 & 26 & 69 & 21 & 49 & 72 & 53 \\
Job 6 & 84 & 2 & 52 & 95 & 48 & 72 & 47 & 65 & 6 & 25 \\
Job 7 & 46 & 37 & 61 & 13 & 32 & 21 & 32 & 89 & 30 & 55 \\
Job 8 & 31 & 86 & 46 & 74 & 32 & 88 & 19 & 48 & 36 & 79 \\
Job 9 & 76 & 69 & 76 & 51 & 85 & 11 & 40 & 89 & 26 & 74 \\
Job 10 & 85 & 13 & 61 & 7 & 64 & 76 & 47 & 52 & 90 & 45 \\
\hline
\end{tabular}


Table 4: Assumptions for the ft10 with maintenance instance

\begin{tabular}{lcc}
\hline Numbers per Machine & Assumption & Value \\
\hline Production Jobs & $f t 10$ & 10 \\
Makespan & $f t 10$ & $930 \mathrm{~h}$ \\
Average Number of Failures & $5 \%$ of production jobs & 0.5 \\
Duration of a failure & $10 \%$ of makespan & $100 \mathrm{~h}$ \\
Available Maintenance & $10 \%$ of production & 1 \\
Actions & jobs & \\
$\begin{array}{lc}\text { Duration of a Maintenance } \\
\text { Actions }\end{array}$ & $2.5 \%$ of makespan & $25 \mathrm{~h}$ \\
\hline
\end{tabular}

Table 5: OSSE of the ft10 with maintenance instance

\begin{tabular}{lllllllllll}
\hline Job & $\delta_{i 1}$ & $\delta_{i 2}$ & $\delta_{i 3}$ & $\delta_{i 4}$ & $\delta_{i 5}$ & $\delta_{i 6}$ & $\delta_{i 7}$ & $\delta_{i 8}$ & $\delta_{i 9}$ & $\delta_{i 10}$ \\
\hline
\end{tabular} \begin{tabular}{lllllllllll}
\hline Job 1 & 0.03 & 0.09 & 0.10 & 0.03 & 0.04 & 0.10 & 0.06 & 0.05 & 0.04 & 0.02
\end{tabular} $\begin{array}{lllllllllll}\text { Job } 2 & 0.03 & 0.08 & 0.06 & 0.10 & 0.08 & 0.03 & 0.04 & 0.05 & 0.08 & 0.02\end{array}$ $\begin{array}{lllllllllll}\text { Job } 3 & 0.08 & 0.09 & 0.03 & 0.09 & 0.11 & 0.10 & 0.10 & 0.08 & 0.03 & 0.03\end{array}$ $\begin{array}{lllllllllll}\text { Job } 4 & 0.06 & 0.08 & 0.08 & 0.12 & 0.10 & 0.04 & 0.07 & 0.10 & 0.02 & 0.03\end{array}$ $\begin{array}{lllllllllll}\text { Job } 5 & 0.10 & 0.10 & 0.02 & 0.06 & 0.02 & 0.07 & 0.10 & 0.05 & 0.07 & 0.05\end{array}$ $\begin{array}{lllllllllll}\text { Job } 6 & 0.07 & 0.10 & 0.05 & 0.09 & 0.04 & 0.06 & 0.04 & 0.08 & 0.10 & 0.02\end{array}$ $\begin{array}{lllllllllll}\text { Job } 7 & 0.03 & 0.03 & 0.07 & 0.10 & 0.03 & 0.02 & 0.03 & 0.07 & 0.02 & 0.05\end{array}$ $\begin{array}{lllllllllll}\text { Job } 8 & 0.02 & 0.11 & 0.04 & 0.09 & 0.02 & 0.07 & 0.10 & 0.04 & 0.04 & 0.09\end{array}$ $\begin{array}{lllllllllll}\text { Job } 9 & 0.06 & 0.06 & 0.06 & 0.04 & 0.10 & 0.10 & 0.05 & 0.07 & 0.02 & 0.06\end{array}$ \begin{tabular}{lllllllllll} 
Job 10 & 0.09 & 0.10 & 0.06 & 0.10 & 0.06 & 0.07 & 0.03 & 0.05 & 0.07 & 0.04 \\
\hline
\end{tabular}

\section{A PROPOSED SOLUTION AND SimULATION APPROACH WITH NEW OPTIMIZATION OBJECTIVE}

Given the new OSSEs, the reliability and the hazard function as well as the thereby formulated MIJSSP, it is now possible to develop new integrated production and maintenance scheduling approaches. Varieties of new optimization goals for these schedules are conceivable. To explore this potential, a GA with the most suitable representation, the job-based representation with repetition [30] is implemented, a new exemplary objective function $C_{\max }{ }^{R}$ is introduced and validated in a simulation setting. The GA is initialized with the random job selection method [31] and uses tournament selection, the PPX crossover operator and insertion and shift mutation [32] at a rate of $95 \%$ and $5 \%$ respectively. The stopping criterion is set to 100 generations.

As shown in literature, the makespan $C_{\max }$ is a suitable benchmark criterion, because it indicates an efficient usage of the production system [15]. To account for the machines' deterioration however, a new minimization objective value is suggested: the Reliability Weighted Makespan $C_{\max }{ }^{R}$. It is defined as the latest reliability weighted completion time

$$
C_{\max }^{R}=\max \left(C_{1}^{R}, C_{2}^{R}, C_{3}^{R}, \ldots, C_{n}^{R}\right)
$$

where $C_{i}^{R}$ equals the completion time $C_{i}$ of Job $J_{i}$ multiplied by $R(t)$ (i.e. how probable the job can be completed without the machine failing) plus a time penalty $F$, weighted according to the failure probability $F(t)=1-R(t)$ :

$$
C_{i}^{R}=R(t) \cdot C_{i}+(1-R(t)) \cdot\left(C_{i}+F\right)
$$

A simulation model is introduced that uses a feasible schedule in the job-based or operation-based representation by [30,32]. It follows the MIJSSP formulation in Chapter IV.B and simulates the machine deterioration according to the reliability prediction framework introduced in Chapter III.B. It calculates the failure probability of each machine, dependent of its deterioration state and current operation specific stress load. Proportional to the calculated probability, it simulates unplanned machine breakdowns according to the failure probability that interrupt the production process of the machine and thus delay the schedule. In this way, the simulation model outputs one or more simulation instances of a given schedule for a generated MIJSSP. The simulation instances contain the actual realized operation schedule with all start and completion times, the occurred failures, the shapes of the machines' deterioration curves as well as their failure probability functions over time. These simulation instances act as the input for the final evaluation. Besides comparing the calculated performances, it is possible to compare different scheduling methods considering different evaluation criteria based on the actual simulation results. A selection of possible criteria includes, but is not limited to: calculated reliability weighted makespan $C_{\max }{ }^{R}$, calculated makespan $C_{\max }$, actual simulated average makespan $C_{\text {sim }}$, average number of failures, cumulated failure probability, total costs and robustness of the schedule.

The results show that the schedule's actual realized average makespan $C_{s i m}$ can differ significantly from the traditionally often calculated makespan $C_{\max }$ e.g. due to a high number of generated machine failures. This simulative evaluation of different scheduling models aims at creating a more realistic assessment of a solution's quality and robustness.

\section{VALIDATION AND RESULTS}

The results allowed for tests and an exemplary validation of the proposed solution method for the introduced problem formulation. The developed GA was tested on the presented MIJSSP benchmark instances $f t 10$. The FIFO (first in first out) heuristic was selected as baseline (I) and an optimal production schedule by [30] and [28] was chosen as the literature benchmark (II) for the performance of the proposed GA (III). Furthermore, the ability of the proposed GA to integrate a PdM action interval for each machine and the result is shown (IV). For the simulation and evaluation 25 simulation runs of each obtained schedule (I-IV) were conducted under the assumptions of constant failure rates $(\beta=1.0)$ and a time penalty $F=200$ for the reliability weighted makespan. Table 6 shows the results of the performance benchmark. The results clearly indicate that the three approaches (II, III, IV) outperform the baseline schedule. The proposed GA cuts down the average number of occurred failures $(4.56<4.68)$. 
Table 6: Results of the performance benchmark

\begin{tabular}{|c|c|c|c|c|c|}
\hline & $\mathrm{C}_{\max }{ }^{\mathrm{R}}$ & $\mathrm{C}_{\max }$ & $\mathrm{C}_{\text {sim }}$ & Robustness & $\overline{\text { Failures }}$ \\
\hline $\begin{array}{l}\text { Baseline FIFO } \\
\text { (I) }\end{array}$ & 2275.7 & 1319 & 1457.6 & $90,5 \%$ & 4.88 \\
\hline $\begin{array}{l}\text { Literature } \\
\text { Benchm. (II) }\end{array}$ & 1701.6 & 930 & 1159.6 & $80,2 \%$ & 4.68 \\
\hline $\begin{array}{l}\text { Proposed GA } \\
\text { (III) }\end{array}$ & 1600.4 & 1107 & 1233.6 & $89,7 \%$ & 4.56 \\
\hline $\begin{array}{l}\text { Proposed GA } \\
\text { w/ PdM action } \\
\text { (IV) }\end{array}$ & 1504.2 & 1125 & 1204.7 & $93,4 \%$ & 3.96 \\
\hline
\end{tabular}

This leads to a lower divergence between the actual realized makespan from the calculated makespan, i.e. enabling a robust schedule. Here, the robustness is calculated by $\mathrm{C}_{\max } / \mathrm{C}_{\text {sim }}$ and represents the divergence of the calculated and the simulated schedule. Robust schedules are prone to random machine failure and maintenance actions and thus give the production planner high planning security. While the proposed GA is able to approximate the optimal solution $C_{\max } \leq 1.15 C_{\max \text {,opt }}$, it did not accomplish to find the best production schedule $C_{\max }$ possible. However, when it comes to maintenance integrated scheduling, the algorithm succeeded to minimize the number of machine failures as opposed to the competing approaches and shows a significant increase in robustness $(89.7 \%>80.2 \%)$.

Instance IV shows that the average performance (1204.7), number of failures (3.96) and robustness (93.4\%) is further improved when introducing the possibility to schedule one PdM action per machine as assumed in Table 5. Fig. 4 depicts the resulting schedule of one simulation run each for the benchmark instances II, III and IV. For the reason of clarity, only machine M4 is presented. The figure shows how $R(t)$ resets to 1 after a maintenance action or failure due to the as good as new assumption for part replacements was scheduled. Furthermore, it can be seen that $R(t)$ retains a high level $(>0.75)$ throughout the whole schedule while applying IV, which clearly highlights the value of approach IV. The model thus enables an optimization of predictive maintenance times with regards to the estimated deterioration and predicted machine reliability under any given objective function.

\section{CONCLUSION}

An extensive literature review in the field of scheduling with PdM information identified a lack of integrated PdM and production scheduling models for the JSSP. Therefore, a theoretical framework to model and integrate PdM information was introduced. The model distinguishes between machine deterioration and failure probability. The deterioration was estimated based on the new Operation Specific Stress Equivalents, while the latter was predicted with the help of the two-parameter Weibull distribution. The reliability function is used to formulate a new Maintenance Integrated Job Shop Scheduling Problem.

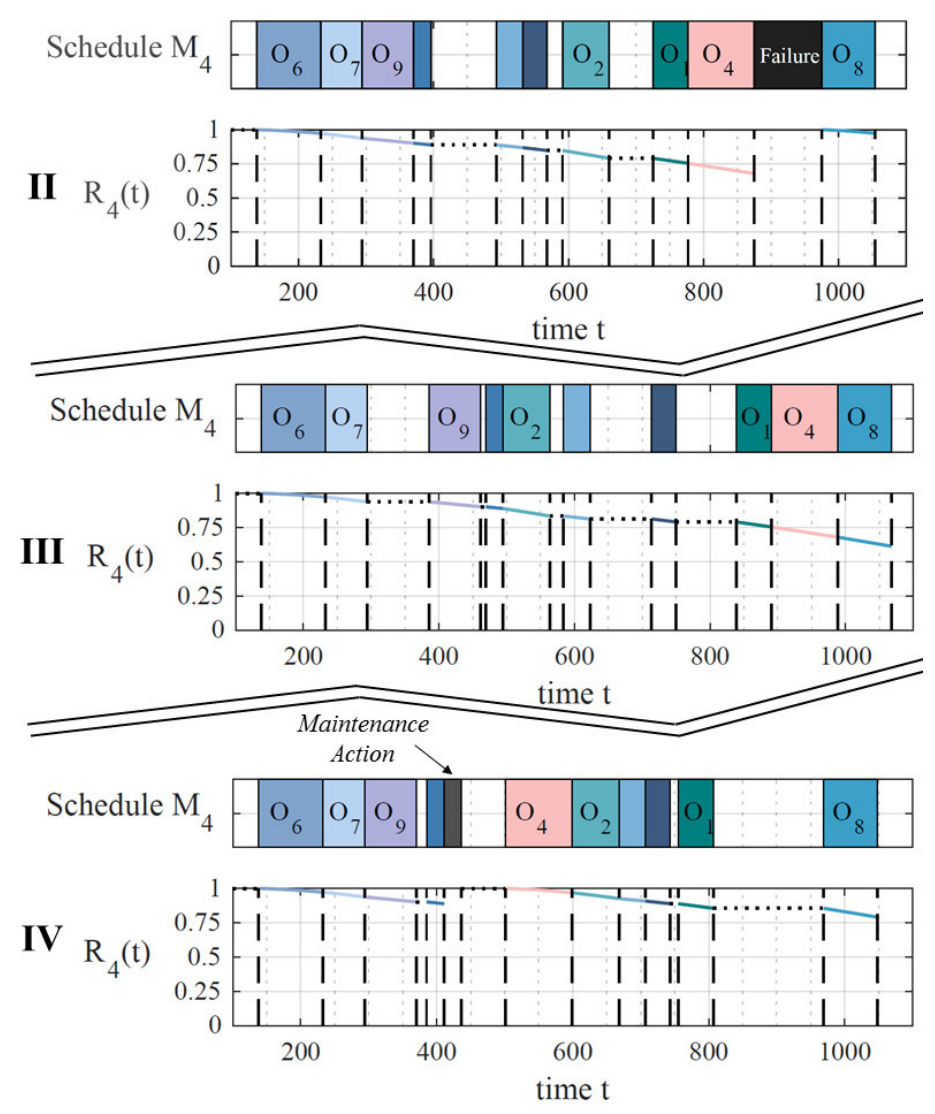

Fig. 4: Resulting schedule for M4 for benchmark instances II-IV.

The formulation fully integrates maintenance actions in the scheduling optimization problem and suggests different possible objective functions. One of which is the reliability weighted makespan $C_{\max }{ }^{R}$ that takes the makespan as well as the machines' estimated reliability into account and subsequently penalizes high failure probabilities. The developed conceptual framework adds to the state of research by proposing the operation specific stress indicator OSSE, applying it in an adopted MIJSSP and evaluating the solution with the help of the new $C_{\max }{ }^{R}$. Simulation runs allowed for a validation that indicated that the proposed algorithm performs better than the basic heuristics across all benchmark instances. However, potential new objective functions not limited to a fixed time penalty parameter are subject to future research.

\section{FUTURE RESEARCH}

The whole model or particular methods and frameworks presented allow future research to further drive the applicability of PdM information in a holistic scheduling approach in the industry practice.

The chosen modelling approach is a static one for now, meaning all jobs and their characteristics are expected to be known in the beginning of the scheduling horizon. The schedule is determined once in the beginning of a production cycle and is pursued regardless of any event happening along the way. In industry practice, dynamic adjustments seem to be realistic and desirable. However, as a first step, a semi-dynamic behavior can be emulated by restarting the proposed scheduling model 
every time the framework conditions have changed. Furthermore, the framework should be extended to cover sequence specific deterioration, e.g. that machine deterioration under high operational speed firstly and then under lower speed could be different than vice versa.

The next and potentially most promising aspect for future research is the further examination of the information required to run the proposed model in the industry practice. In this context, the model parameters as well as the desired operation specific inputs must be approximated with the help of historic and real-time data. New analytics methods such as artificial neural networks might allow for a promising dynamic feedback loop from the shop floor to deterioration estimation and failure prediction. Real-time data insights also enable a dynamically predicting and reacting scheduling model. Furthermore, monetary aspects can be included in the model in order to derive the cost-optimal production schedule. This opens the door to completely new maintenance policies as well as holistic production strategies. In this context, Predictive Maintenance might be the enabler for a dynamic maintenance-integrated production scheduling process [33] and will play a significant role in the future success of Industrie 4.0.

\section{ACKNOWLEDGMENT}

This research was conducted in relation to the Predictive Cognitive Maintenance Decision Support System (PreCoM) project under the Grant Agreement No. 768575, funded by European Union's Horizon 2020 program.

\section{REFERENCES}

[1] X.-S. Si, W. Wang, C.-H. Hu, and D.-H. Zhou, "Remaining useful life estimation - A review on the statistical data driven approaches," European Journal of Operational Research, vol. 213, no. 1, pp. 1-14, 2011.

[2] S. Feldmann, O. Herweg, H. Rauen, and P.-M. Synek, "Predictive Maintenance: Service der Zukunft - und wo er wirklich steht," München, 2017.

[3] S. AG, "STAUFEN. Industrie 4.0 Index 2017,"

[4] L. A. Hadidi, U. M. A. Turki, and A. Rahim, "Integrated models in production planning and scheduling, maintenance and quality: a review," IJISE, vol. 10, no. 1, p. 21, 2012.

[5] A. El Khalfi, S. Aarab, and A. El Barkany, "The integration of maintenance plans and production scheduling taking account of outsourcing: a literature review," IJPQM, vol. 21, no. 1, p. 1, 2017.

[6] R. Gouriveau and K. Medjaher, "Prognostics. Part : Industrial Prognostic - An Overview," in Maintenance modelling and applications, J. Andrews, C. Bérenguer, and L. Jackson, Eds., Høvik, Norway: DNV Det Norske Veritas, 2011, pp. 10-30.

[7] Q. Li, Z. Gao, D. Tang, and B. Li, "Remaining useful life estimation for deteriorating systems with time-varying operational conditions and condition-specific failure zones," Chinese Journal of Aeronautics, vol. 29, no. 3, pp. 662-674, 2016.

[8] R. Gao et al., "Cloud-enabled prognosis for manufacturing," CIRP Annals, vol. 64, no. 2, pp. 749-772, 2015.

[9] A. Heng, S. Zhang, A. C.C. Tan, and J. Mathew, "Rotating machinery prognostics: State of the art, challenges and opportunities," Mechanical Systems and Signal Processing, vol. 23, no. 3, pp. 724-739, 2009.

[10] E. Ramasso and A. Saxena, "Review and Analysis of Algorithmic Approaches Developed for Prognostics on CMAPSS Dataset," Annual Conference of PHM Society, vol. 2014.
[11] L. Bian, N. Gebraeel, and J. P. Kharoufeh, "Degradation modeling for real-time estimation of residual lifetimes in dynamic environments," IIE Transactions, vol. 47, no. 5, pp. 471-486, 2015.

[12] H. Liao and Z. Tian, "A framework for predicting the remaining useful life of a single unit under time-varying operating conditions," IIE Transactions, vol. 45, no. 9, pp. 964-980, 2013.

[13] acatech, Ed., "Smart Maintenance für Smart Factories: Mit intelligenter Instandhaltung die Industrie 4.0 vorantreiben," 2015.

[14] B. Naderi, M. Zandieh, and S. M. T. Fatemi Ghomi, "Scheduling sequence-dependent setup time job shops with preventive maintenance," The International Journal of Advanced Manufacturing Technology, vol. 43, no. 1-2, pp. 170-181, 2009.

[15] M. L. Pinedo, Ed., Scheduling: Theory, Algorithms, and Systems, 5th ed. Cham, Heidelberg, New York, Dordrecht, London: Springer, 2016

[16] L. A. Hadidi, U. M. A. Turki, and M. A. Rahim, "Joint job scheduling and preventive maintenance on a single machine," IJOR, vol. 13, no. 2, p. 174, 2012.

[17] X. Chen, L. Xiao, X. Zhang, W. Xiao, and J. Li, "An integrated model of production scheduling and maintenance planning under imperfect preventive maintenance," Maintenance and Reliability, vol. 17, no. 1, pp. 70-79, 2015.

[18] N. Fnaiech, C. Fitouri, C. Varnier, F. Fnaiech, and N. Zerhouni, “A New Heuristic Method for Solving Joint Job Shop Scheduling of Production and Maintenance,"

[19] H. Beheshti-Fakher, M. Nourelfath, and M. Gendreau, "Joint planning of production and maintenance in a single machine deteriorating system,"

[20] C. Fitouri, N. Fnaiech, C. Varnier, F. Fnaiech, and N. Zerhouni, "A Decison-Making Approach for Job Shop Scheduling with Job Depending Degradation and Predictive Maintenance,"

[21] A. Ladj, C. Varnier, and F. B.-S. Tayeb, "IPro-GA: An integrated prognostic based GA for scheduling jobs and predictive maintenance in a single multifunctional machine," IFAC-PapersOnLine, vol. 49, no. 12, pp. 1821-1826, 2016.

[22] M. A. Noman, E. S. A. Nasr, A. Al-Shayea, and H. Kaid, "Overview of predictive condition based maintenance research using bibliometric indicators," Journal of King Saud University - Engineering Sciences, 2018.

[23] J. F. Lawless, Statistical models and methods for lifetime data, 2nd ed. Hoboken, N.J.: Wiley-Interscience, 2003.

[24] M. Strunz, Instandhaltung: Grundlagen - Strategien - Werkstätten. Berlin, Heidelberg: Springer Vieweg, 2012.

[25] Fisher, H., Thopmspon, G., "Probabilistic learning combinations of local job-shop scheduling rules," Industrial Scheduling, 1963.

[26] J. Adams, E. Balas, and D. Zawack, "The Shifting Bottleneck Procedure for Job Shop Scheduling," Management Science, vol. 34, no. 3, pp. 391-401, 1988.

[27] D. Applegate and W. Cook, "A Computational Study of the Job-Shop Scheduling Problem," ORSA Journal on Computing, vol. 3, no. 2, pp. 149-156, 1991.

[28] J. J. van Hoorn, "The Current state of bounds on benchmark instances of the job-shop scheduling problem," J Sched, vol. 21, no. 1, pp. 127 $128,2018$.

[29] E. Taillard, "Benchmarks for basic scheduling problems," European Journal of Operational Research, vol. 64, no. 2, pp. 278-285, 1993.

[30] T. Yamada, Nakano, and R., "Jop-shop scheduling," in IEE control engineering series, vol. 55, Genetic algorithms in engineering systems, A. M. S. Zalzala and P. J. Fleming, Eds., London: Institution of Electrical Engineers, 1997, pp. 134-160.

[31] V. S. Jorapur, V. S. Puranik, A. S. Deshpande, and M. Sharma, "A Promising Initial Population Based Genetic Algorithm for Job Shop Scheduling Problem," JSEA, vol. 09, no. 05, pp. 208-214, 2016.

[32] F. Werner, "A Survey of Genetic Algorithms for Shop Scheduling Problems," Heuristics: Theory and Applications, pp. 161-222, 2013.

[33] S. Zhai and G. Reinhart, "Predictive Maintenance als Wegbereiter für die instandhaltungsgerechte Produktionssteuerung" $Z W F$, vol. 113, no. 5, pp. 298-301, 2018. 